\title{
XỬ LÝ PHỔ ẢNH VỆ TINH VNREDSAT-1
}

\author{
NGUYẼ̃N TH!̣ MAI DUNG ${ }^{(1)}$, NGUYẼ̃N TRƯỜNG XUÂN(1), \\ TRẦN XUÂN TRƯỜNG ${ }^{(1)}$, TRẦ MAI HƯO'NG(1) \\ LƯU VĂN DOANH ${ }^{(2)}$ \\ (1) Trường Đại học Mỏ - Địa chất \\ (2) Sở Tài nguyên và Môi trường Phú Thọ
}

\section{Tóm tắt:}

Xử lý phổ hay hiệu chỉnh phổ là quá trình loại bỏ hoặc làm giảm bót các sai số do ảnh hưởng của điều kiện khí quyển, nguồn sáng chiếu và bề mặt địa hình. Có hai loại hiệu chỉnh phổ: hiệu chỉnh tuyệt đối và hiệu chỉnh tương đối. Trong bài báo nhóm nghiên cứu tập trung tìm hiểu các phương pháp hiệu chỉnh phổ tương đối từ đó xây dựng phương pháp hiệu chỉnh phổ trên ảnh vệ tinh VNREDSat-1. Phương pháp được lựa chọn bao gồm nắn chỉnh hình học ảnh, lựa chọn các đối tượng bất biến giả định, xác định tham số chuẩn hóa. Kết quả thực nghiệm được kiểm định qua các phép phân tích thống kê giá trị độ sáng của pixel trên ảnh trước và sau chuẩn hóa phổ. Độ chính xác của kết quả thể hiện phương pháp lựa chọn là hợp lý.

\section{Mở đầu}

Trong các nghiên cứu đánh giá biến động sử dụng đất và lớp phủ đất, nguồn dữ liệu quan trọng là các tư liệu ảnh vệ tinh. Tuy nhiên chất lượng của các dữ liệu này thường phụ thuộc vào điều kiện ghi nhận hình ảnh, bộ cảm, địa hình... Ngoài ra sự che phủ của các đám mây cũng là nguyên nhân dẫn đến những sai lệch về giá trị phản xạ phổ trên ảnh vệ tinh.

Xử lý phổ hay còn gọi là hiệu chỉnh phổ trên ảnh vệ tinh là quá trình loại bỏ hoặc làm giảm bớt sự khác biệt giữa giá trị phổ thu nhận được bằng bộ cảm và giá trị phản xạ phổ cũng như bức xạ phổ của các đối tượng thực trên mặt đất, và thường chia làm hai loại là hiệu chỉnh tuyệt đối và hiệu chỉnh tương đối. Hiệu chỉnh phổ tuyệt đối bao gồm hiệu chỉnh các sai số phổ do ảnh hưởng của bộ cảm, nguồn chiếu sáng, hiện tượng phân tán và hấp thụ trong khí quyển,... Các phương pháp chủ yếu thường được sử dụng bao gồm hiệu chỉnh

Ngày nhận bài: 18/4/2016 giá trị phổ về giá trị chuẩn dựa vào tham số chuyển đổi khí quyển, đường cong phản xạ phổ tại các phòng thí nghiệm, các đối tượng đen tuyệt đối,... Tuy nhiên trên thực tế việc hiệu chỉnh tuyệt đối các giá trị phổ thường rất phức tạp do kinh phí cao cũng như rất khó thu nhận các thông số khí quyển và mặt đất tại đúng thời điểm ghi nhận ảnh. Hiệu chỉnh phổ tương đối là phương pháp chuẩn hóa giá trị phản xạ phổ hay các giá trị số (Digital Numbers - DN) trên từng kênh ảnh của các ảnh vệ tinh đa thời gian theo một ảnh lựa chọn trước (ảnh tham chiếu hay ảnh gốc) (Yuan, Elvidge, 1996). Hiệu chỉnh phổ tương đối không yêu cầu các tham số khí quyển, thông số của thiết bị ghi nhận hình ảnh, ngoài ra còn sử dụng các thuật toán đơn giản nên thường được sử dụng rộng rãi. Ảnh kết quả sau khi chuẩn hóa sẽ có cùng đặc trưng về khí quyển, tia sáng chiếu tới như tại thời điểm ghi nhận ảnh gốc (Hall, 1991). Một số nghiên cứu về phương pháp hiệu chỉnh phổ tương đối đã được công bố như Schott, 1988; Eckhardt, 1990;

Ngày chấp nhận đăng: 10/5/2016 
Hall, 1991; Elvidge, 1995; Yuan và Elvidge, 1996; Heo và FitzHugh, 2000; Yang và Lo, 2000, và có thể phân loại thành các nhóm: hiệu chỉnh theo phương pháp thống kê, tương quan biểu đồ phân bố giá trị độ xám, và chuẩn hóa theo hàm hồi quy tuyến tính.

Trong bài báo này, nhóm nghiên cứu tập trung tìm hiểu, đánh giá các phương pháp hiệu chỉnh phổ tương đối từ đó xây dựng phương pháp hiệu chỉnh phổ phù hợp cho tư liệu ảnh vệ tinh VNREDSat-1. Phương pháp hiệu chỉnh được xây dựng sẽ được kiểm định trên ảnh vệ tinh VNREDSat -1 khu vực tỉnh Phú Thọ.

\section{Xử lý phổ ảnh vệ tinh VNREDSat-1}

Để phục vụ cho bài toán phân tích và đánh giá biến động, các tư liệu ảnh vệ tinh VNREDSat-1 cần phải được hiệu chỉnh các sai số do ảnh hưởng của điều kiện khí quyển, góc chiếu sáng và địa hình. Bài báo này chỉ tập trung nghiên cứu phương pháp hiệu chỉnh phổ tương đối ảnh vệ tinh VNREDSat-1.

Quá trình xử lý phổ bao gồm các bước chính: nắn chỉnh hình học ảnh, lựa chọn các đối tượng bất biến giả định, xác định tham số chuẩn hóa.

\section{A. Tiền xử lý: Nắn chỉnh hình học ảnh.}

Ảnh toàn sắc và ảnh đa phổ của ảnh vệ tinh VNREDSat-1 được nắn chỉnh hình học hay được chuyển đổi về cùng hệ quy chiếu với ảnh tham chiếu trước khi tiến hành bước hiệu chỉnh phổ tương đối.

B. Lựa chọn các đối tượng bất biến giả định (Pseudo-invariant Features - PIFs)

Phương pháp hiệu chỉnh phổ tương đối được xây dựng trong bài báo được thực hiện dựa trên giả thiết các đối tượng bất biến giả định PIFs là các đối tượng được xác định trên ảnh và không bị ảnh hưởng bởi các yếu tố chu kỳ thời tiết, môi trường. Các đối tượng này có thể là các đối tượng có mầu sáng trên ảnh như các đô thị, các đối tượng có cấu trúc bê tông, đá... hoặc là các đối tượng có mầu đen như các khu vực nước sâu trên ảnh.

Có nhiều phương pháp lựa chọn PIFs đã được phát triển, tuy nhiên dựa vào việc đánh giá ưu nhược điểm của từng phương pháp cũng như phân tích các đặc điểm của ảnh vệ tinh VNREDSat-1, nhóm nghiên cứu lựa chọn phương pháp đánh giá biến động đa chiều (Multivariate Alteration Detection MAD). Phương pháp này được phát triển bởi Neilsen, (1998) và được phát triển thêm bởi Canty (2008). Phương pháp dựa trên sự phân tích tương quan chuẩn (Canonical Correlation Analysis - CCA).

Giả sử ta có hai dữ liệu ảnh đầu vào với $\mathrm{N}$ kênh phổ, giá trị độ sáng trên hai tấm ảnh được định nghĩa bởi vector $F$ và $G$, trong đó:

$$
\begin{aligned}
& F=\left[F_{1}, F_{2}, F_{3}, \ldots F_{N}\right]^{\top} \\
& G=\left[G_{1}, G_{2}, G_{3}, \ldots G_{N}\right]^{\top}
\end{aligned}
$$

Tổ hợp tuyến tính giá trị độ sáng trên tất cả các kênh ảnh được tính theo công thức:

$$
\begin{gathered}
U=a^{\top} F=\left[a_{1} F_{1}, a_{2} F_{2}, a_{3} F_{3}, \ldots a_{N} F_{N}\right]^{\top} \\
G=b^{T} F=\left[b_{1} F_{1}, b_{2} F_{2}, b_{3} F_{3}, \ldots b_{N} G_{N}\right]^{\top}
\end{gathered}
$$

Sự khác biệt giữa các tổ hợp tuyến tính $U$ và $V$ được định nghĩa là các biến ngẫu nhiên của MAD. Giá trị khác biệt cực đại tương ứng hàm tương quan giữa $U$ và $V$ đạt giá trị nhỏ nhất, nghĩa là $\operatorname{Var}(\mathrm{U})=\operatorname{Var}(\mathrm{V})=$ 1.

Sử dụng phân tích tương quan chuẩn $\mathrm{CCA}$, xác định tổ hợp tuyến tính $\mathrm{U}=\mathrm{a}^{\top} \mathrm{F}$ và $\mathrm{V}=\mathrm{b}^{\top} \mathrm{G}$ của các biến phân bố chuẩn Gauss $|U, V| \in N(\mu, \Sigma)$ với giá trị tương quan lớn nhất:

$$
\rho=\operatorname{Corr}[U, V]=\frac{\operatorname{cov}[U, V]}{\sqrt{\operatorname{var}[U] \operatorname{var}[V]}}=\frac{a^{T} \Sigma_{1,2} b}{\sqrt{a^{T} \Sigma_{1,1} a} \sqrt{b^{T} \Sigma_{2,2} b}}
$$


Ta có:

$$
\begin{aligned}
& \sum_{F G} \sum_{G G}^{-1} \sum_{G F} a=\rho^{2} \sum_{F F} a \\
& \sum_{G F} \sum_{F F}^{-1} \sum_{F G} b=\rho^{2} \sum_{G G} b
\end{aligned}
$$

Hàm chuyển đổi của MAD được định nghĩa là hiệu của các biến tương quan chuẩn. Các biến của MAD là không tương quan lẫn nhau, thành phần cuối cùng có giá trị tương quan lớn nhất tương ứng với trị số khác biệt nhỏ nhất và thành phần đầu tiên sẽ có trị số khác biệt lớn nhất.

Hai tấm ảnh thu nhận ở các điều kiện khí quyển khác nhau với cùng một lớp phủ đất sẽ có trị số khác biệt tuân theo sự phân bố chuẩn Gauss. Cụ thể, các biến của MAD, thường không tương quan, sẽ tuân theo luật phân bố chuẩn với ma trận đường chéo hiệp phương sai.

Trong trường hợp một vài lớp phủ có sự thay đổi, sự phân bố sẽ lệch đi so với phân bố chuẩn Gauss.

Các pixel không thay đổi được phân biệt với các pixel thay đổi dựa trên ngưỡng quyết định. Tổng của bình phương độ lệch chuẩn của các biến $M A D$ được xác định theo công thức (Hardoon, 2003):

$$
Z=\sum_{i=1}^{N}\left(\frac{M_{i}}{\sigma_{M_{i}}}\right)^{2}
$$

Trong đó $\sigma_{M_{i}}^{2}=2\left(1-\rho_{N-i+1}\right)$

Do đó khi các biến không thay đổi tuân theo phân bố chuẩn và không tương quan, các biến ngẫu nhiên $Z$ sẽ có phân bố $\mathrm{CHI}$ squared với $\mathrm{N}$ bậc (hàm phân bố $P_{\chi^{2},{ }^{2}\left({ }^{(z)}\right)}$ ). Trọng số xác định bởi hàm phân bố $\mathrm{CHI}$ square sử dụng để tính các biến $z$ được tính theo công thức:

$$
\operatorname{Pr}(\text { no change })=1-P_{\chi^{2} ; N}(z)
$$

$\operatorname{Pr}$ (no change) là xác suất một pixel $Z$ được xác định từ hàm phân bố $\mathrm{CHI}$-squared có thể lớn hoặc rất lớn. $Z$ có giá trị nhỏ tương ứng với xác suất không thay đổi là lớn.

Vòng lặp của phép chuyển đổi MAD sẽ kết thúc khi đạt được các điều kiện như không có đủ sự thay đổi trong hàm tương quan chính

Với mục đích chuẩn hóa phổ, các pixel được lựa chọn khi giá trị $\operatorname{Pr}$ (no change) lớn hơn ngưỡng quyết định $\mathrm{t}$, thường là $95 \%$.

Hình 1, 2 và 3 mô tả sự tuyến tính của các PIFs cho mỗi kênh ảnh. Giá trị pixel nhỏ hơn ngưỡng $t$ được hiểu là các PIFs trên các kênh tương ứng.



Hình 1: Đồ thị của PIFs cho kênh 1



Hình 2: Đồ thị của PIFs cho kênh 2 


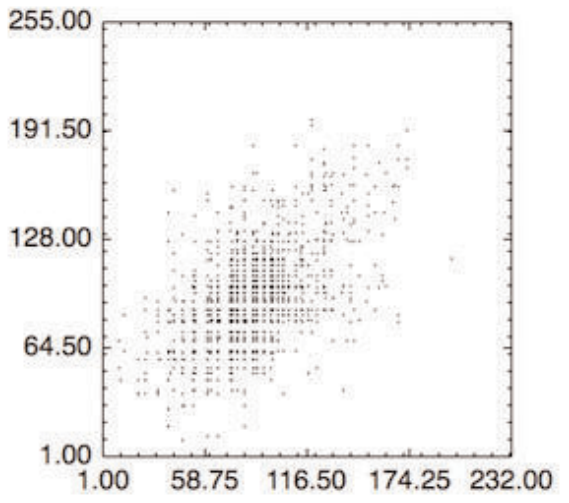

Hình 3: Đồ thị của PIFs cho kênh 3

\section{Kết quả}

Ảnh vệ tinh VNREDSat-1 khu vực nghiên cứu chụp tại hai thời điểm khác nhau được lựa chọn tiến hành thực nghiệm. Các dữ liệu ảnh này đều được nắn chỉnh về hệ tọa độ VN2000.

Phương pháp xử lý phổ MAD được áp dụng cho ảnh thực nghiệm. Kết quả của quả trình phân tích tương quan chuẩn (CCs) được thể hiện qua đồ thị 3.1.

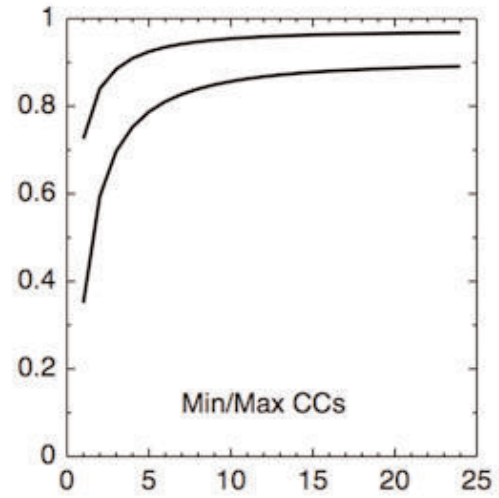

Hình 4: Kết quả phân tích tương quan chuẩn

Độ chính xác và độ tin cậy của bước xử lý phổ được kiểm chứng thông qua đánh giá giá trị trung bình và phương sai của ảnh trước và sau khi hiệu chỉnh phổ cũng như tính toán thống kê hệ số tương quan tương quan $R^{2}$ của giá trị độ sáng của các pixel trên hai tấm ảnh.
Bảng 3.1. Kết quả đánh giá hệ số tương quan của ảnh sau hiệu chuẩn phổ.

Regression Coefficients (528 Points)

\begin{tabular}{|c|c|c|c|}
\hline Band & Slope & Intercept & Correlation \\
\hline 1 & 0.946 & -5.047 & 0.990 \\
\hline 2 & 0.920 & 0.760 & 0.985 \\
\hline 3 & 0.856 & 2.317 & 0.995 \\
\hline
\end{tabular}

Hình 6 minh họa ảnh vệ tinh VNREDSat1 khu vực thực nghiệm tại hai thời điểm ghi nhận ảnh năm 2014, 2015, bao gồm ảnh gốc (ảnh tham chiếu), ảnh hiệu chỉnh và ảnh kết quả sau khi chuẩn hóa theo phương pháp MAD.

\section{Kết luận}

Với mục đích xây dựng phương pháp xử lý phổ cho ảnh vệ tinh VNREDSat-1, dựa trên đặc điểm của tư liệu ảnh, bài báo chỉ tập trung nghiên cứu phương pháp hiệu chỉnh phổ tương đối cho ảnh vệ tinh đa phổ. Phương pháp hiệu chỉnh được lựa chọn được áp dụng trên ảnh vệ tinh VNREDSat 1 khu vực tỉnh Phú Thọ. Kết quả được kiểm định qua các phép phân tích thống kê giá trị độ sáng của pixel trên ảnh trước và sau chuẩn hóa phổ. Độ chính xác của ảnh kết quả thể hiện phương pháp được lựa chọn là hợp lý.

Bài báo là một phần kết quả nghiên cứu của đề tài $\mathrm{KHCN}-\mathrm{VT} 12-15$. Qua bài báo nhóm nghiên cứu gửi lời cảm ơn tới Ban Chương trình Khoa học và Công nghệ Vũ trụ 2012 - 2015 vì những định hướng và hỗ trợ quý báu trong suốt thời gian thực hiện đề tài. $O$

\section{Tài liệu tham khảo}

[1]. Canty, M. J., Nielsen, A. A., Schmidt M., 2004. Automatic radiometric normalization of multi-temporal satellite imagery. Remote Sensing f Environment, 91:4411451. 

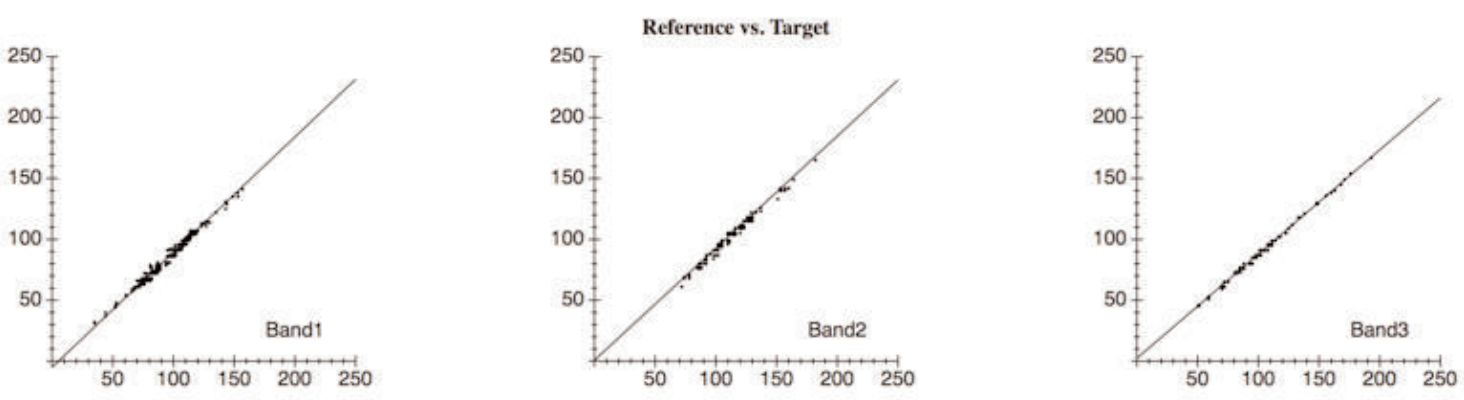

Hình 5: Hàm hồi quy trên ba kênh phổ RGB của ảnh sau xử lý phổ

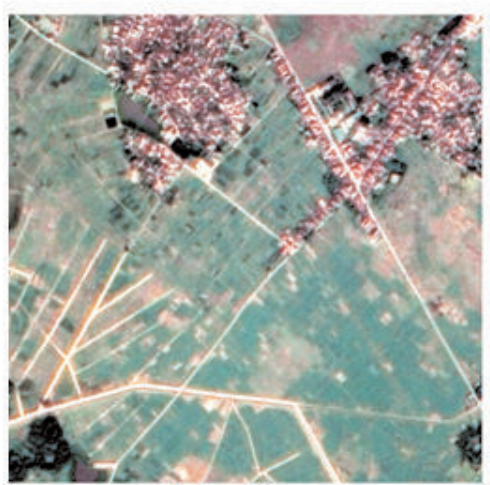

Ảnh gốc

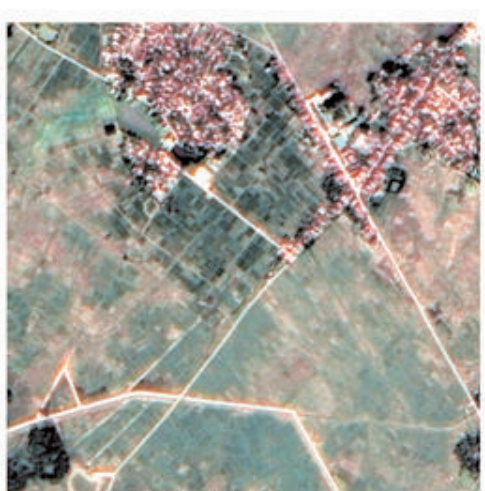

Ảnh đối tượng

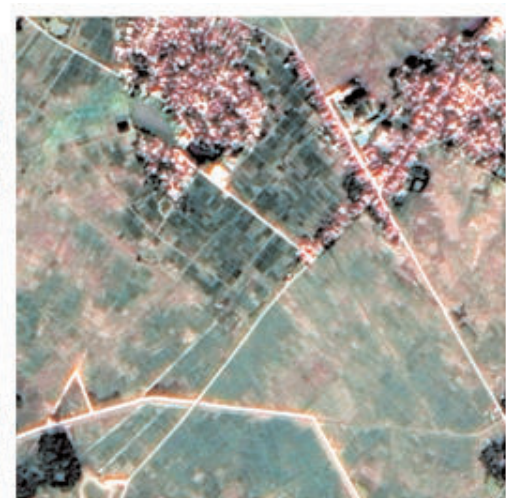

Ảnh chuần hóa

Hình 6: Ảnh vệ tinh VNREDSat-1 khu vực nghiên cứu trước và sau khi xử lý phổ

[2]. Canty, M., Nielsen, A., "Automatic radiometric normalization of multitemporal satellite imagery with the iteratively reweighted MAD transformation". Remote Sensing of Environment, vol 112,issue 3, 1025-1036. 2007.

[3]. Eckhardt, D.W., J.P. Verdin and G.R. Lyford, 1990. Automated update of an irrigated lands GIS using SPOT HRV imagery. Photogramm. Eng. Remote Sens., 56: 1515-1522.

[4]. Elvidge, C.D., D. Yuan, R.D. Werackoon and R.S. Lunetta, 1995. Relative radiometric normalization of landsat Multispectral Scanner (MSS) data using an automated scattergram controlled regression. Photogramm. Eng. Remote Sens., 61: 1255-1260.

[5]. Hall, F.G., D.E. Strebel, J.E. Nickeson and S.J. Goetz, 1991. Radiometric rectification toward a common radiometric response among multidate, multisensor images. Remote Sens. Environ., 35: 11-27.

[6]. Heo, J. and T.W. FitzHugh, 2000. A standardized radiometric normalization method for change detection using remotely sensed imagery. Photogramm. Eng. Remote Sens.,66: 173-181.

[7]. Nielsen, A. A., Conradsen, K., \& Simpson, J. J. (1998). Multivariate alteration detection (MAD) and MAF post-processing in multispectral, bitemporal image data: New approaches to change detection studies. Remote Sensing of Environment, 64, 1-19. I

[8]. Schott, J.R., C. Salvaggio and W.J. Volchok, 1988. Radiometric scene normalization using pseudoinvariant features. 
Remote Sens. Environ., 26: 1-16.

[9]. Yang, X.J. and C.P. Lo, 2000. Relative radiometric normalization performance for change detection from multi-date satellite images. Photogramm.
Eng. Remote Sens., 66: 967-980.

[10]. Yuan, D. and C.D. Elvidge, 1996. Comparison of elative radiometric normalization techniques. ISPRS J. Photogramm. Remote Sens., 51: 117-126.O

\section{Summary}

\section{Radiometric Correction of VNREDSat-1 satellite image}

Nguyen Thi Mai Dung, Nguyen Truong Xuan, Tran Xuan Truong, Tran Mai Huong

Hanoi University of Mining and Geology

\section{Luu Van Doanh, Environment and Natural Resources Department, Phutho province}

Because of the complexity of full remote sensing image correction, there has been considerable interest in image-based techniques that provide relative normalization. Relative radiometric correction is a method of correction that applies one image as a reference and adjusts the radiometric properties of subject image to match the reference. In this paper, we present a radiometric normalization technique to select pseudo invariant features (PIFs) in multispectral satellite image. Multivariate Alteration Detection method (MAD) has been employed for VNREDSat-1 satellite image of the study areas. Results of the experimentation show that MAD provides a fast, reliable and robust method for radiometric normalization of VNREDSAT-1 multispectral image time series. $\mathrm{O}$

\section{GIẢI PHÁP LỰA CHỌN PHƯƠNG PHÁP TRỘN ẢNH....}

(Tiếp theo trang 27)

\section{Summary}

\section{Selecting image fusion methods for hight resolution optical satellite images}

\section{MSc. Do Thi Hoai, Institute of Geodesy and Cartography}

Image fusion means that is a combination technology using types of image to create another image. New image includes more information to enhance the ability of analysis and recognize the features on that. - Combination of panchromatic images with multispectral images (same type of image - optical images) to create new images have high spectral resolution (same as multispectral images) and high spatial resolution (same as panchromatic images). In the article, author presents the results of research selecting image fusion methods for high resolution optical satellite image (WorldView - 2) and Analysis of spectral quality assessment indexes of the image before the fused images. The effectiveness of for techniques, the intensity-hue-saturation (IHS), principal component analysis (PCA), Brovey and Multiplicative have been evaluated through visual inspection and correlation analysis. $O$ 